\title{
Does early surgery 'EASE' IE outcomes?
}

Results of the EASE trial have been published in the New England Journal of Medicine. On the basis of their findings, the EASE investigators suggest "that early surgery is a valuable therapeutic option to prevent embolism" in patients with left-sided infective endocarditis (IE).

The EASE trial was a prospective, randomized study comparing early surgery (within $48 \mathrm{~h}$ of randomization; $n=37$ ) with conventional therapy (as per the AHA guidelines; $n=39$ ) in patients with IE. According to Dr Duk-Hyun Kang, lead author of the report, randomization was mostly performed within 2 days of definite diagnosis of IE. The trial was conducted in the Asan Medical Center and the Seoul National University Hospital in Korea. Enrolled patients were aged $18-80$ years, had severe valvular disease (affecting mitral or aortic valves), but no prosthetic valve, and had left-sided vegetation with diameter $>10 \mathrm{~mm}$, but no right-sided vegetations. The mean age of participants was 47 years.

Early surgery was associated with a substantial reduction in in-hospital death or embolic events within the first 6 weeks (3\% vs $23 \%$ with conventional treatment; HR 0.10, 95\% CI 0.01-0.82, $P=0.03$ ) the primary end point of the trial. The incidence of in-hospital death did not differ between the two groups (1 [3\%] in each group), but the number of embolic events within the first 6 weeks did differ (none of those assigned to early surgery and 8 [21\%] of the conventional-therapy group experienced such events; $P=0.005$ ). Notably, $77 \%$ of patients assigned to conventional therapy underwent valve surgery during the initial hospitalization (69\%) or during follow-up (8\%). However, all primary end points in this group occurred before surgery.

The EASE investigators warn that "the results of our study may not be applicable to low-volume medical centers or to patients with high operative risk."

Bryony M. Mearns

Original article Kang, D.-H. et al. Early surgery versus conventional treatment for infective endocarditis. N. Engl. J. Med. 366, 2466-2473 (2012) 